\title{
Polyoctahedral Silsesquioxane Hexachlorocyclotriphosphazene Membranes for Hot Gas Separation
}

\author{
Farzaneh Radmanesh, ${ }^{\dagger}$ Maria G. Elshof, ${ }^{\dagger}$ and Nieck E. Benes*
}

Cite This: ACS Appl. Mater. Interfaces 2021, 13, 8960-8966

Read Online

ABSTRACT: There is a need for gas separation membranes that can perform at high temperatures, for example, for $\mathrm{CO}_{2}$ capture in industrial processes. Polyphosphazenes classify as interesting materials for use under these conditions because of their high thermal stability, hybrid nature, and postfunctionalization options. In this work, thin-film composite cyclomatrix polyphosphazene membranes are prepared via the interfacial polymerization reaction between polyhedral oligomeric silsesquioxane and hexachlorocyclotriphosphazene on top of a ceramic support. The prepared polyphosphazene networks are highly crosslinked and show excellent thermal stability until $340{ }^{\circ} \mathrm{C}$. Single gas permeation experiments at temperatures ranging from 50 to $250{ }^{\circ} \mathrm{C}$ reveal a

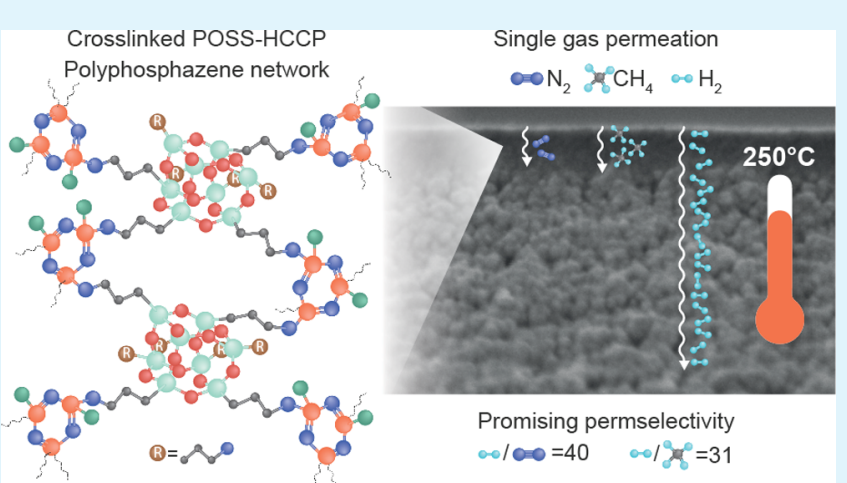
molecular sieving behavior, with permselectivities as high as 130 for $\mathrm{H}_{2} / \mathrm{CH}_{4}$ at the low temperatures. The permselectivities of the membranes persist at the higher temperatures; at $250{ }^{\circ} \mathrm{C} \mathrm{H}_{2} / \mathrm{N}_{2}$ (40), $\mathrm{H}_{2} / \mathrm{CH}_{4}(31) \mathrm{H}_{2} / \mathrm{CO}_{2}(7)$, and $\mathrm{CO}_{2} / \mathrm{CH}_{4}$ (4), respectively, while maintaining permeances in the order of $10^{-7}$ to $10^{-8}$ mol $\mathrm{m}^{-2} \mathrm{~s}^{-1} \mathrm{~Pa}^{-1}$. Compared to other types of polymer-based membranes, especially the $\mathrm{H}_{2} / \mathrm{N}_{2}$ and $\mathrm{H}_{2} / \mathrm{CH}_{4}$ selectivities are high, with similar permeances. Consequently, the hybrid polyphosphazene membranes have great potential for use in high-temperature gas separation applications.

KEYWORDS: gas separation, polyphosphazene, POSS, thermal stability, interfacial polymerization

\section{INTRODUCTION}

Membrane technology can be used for a great variety of separation processes. The gas separation market uses membranes for many molecular separations, such as for the adjustment of the $\mathrm{H}_{2} / \mathrm{CO}-\mathrm{CO}_{2}$ ratio in syngas production, the enrichment of $\mathrm{N}_{2}$, acid gas removal from natural gas, and olefin/paraffin separations. ${ }^{1-7}$ However, at high temperatures, the separation of small gases is more difficult because of limited thermal stability and the increment of molecular dynamics of polymeric membranes. ${ }^{3}$ By improving the stability and performance of polymeric membranes at these high-temperature conditions, they can offer a promising alternative to traditional technologies, such as for $\mathrm{CO}_{2}$ capture and recovery in industrial processes. ${ }^{8}$ Therefore, over the years, research has been focused on developing membranes that can perform the required separations at high temperatures, but the available number of studies is still limited. ${ }^{3,9-11}$ For this, a range of materials have been identified that might be of use for this purpose. $^{12}$

One of the classes of materials of interest for hightemperature applications is polyphosphazenes. ${ }^{12,13}$ These materials are attractive because of their robustness, hybrid nature, and postfunctionalization options. The basis for most polyphosphazenes is hexachlorocyclotriphosphazene (HCCP).
It can be used as a building block, for example, for the formation of supramolecular self-assemblies or crosslinked structures. When HCCP reacts with di- or trifunctional compounds, it forms a cyclomatrix poly(phosphazene) network. ${ }^{14}$ These crosslinked structures are of high interest for their flame-retardant properties and good thermal stability. ${ }^{15,16}$

Over the years, polyphosphazenes have gained interest for the preparation of different types of membranes. For instance, a variety of polyphosphazenes have been used as proton exchange membranes, but they also have found their application as a nanofiltration membrane. ${ }^{17-19}$ Gas separation properties of polyphosphazene membranes have also been studied. $^{20}$ However, in most cases, the linear poly(dichlorophosphazene) has been used as a building block, and the performance has only been studied at temperatures lower than $100{ }^{\circ} \mathrm{C} .{ }^{21}$ The gas separation performance of

Received: December 11, 2020

Accepted: January 29, 2021

Published: February 10, 2021 

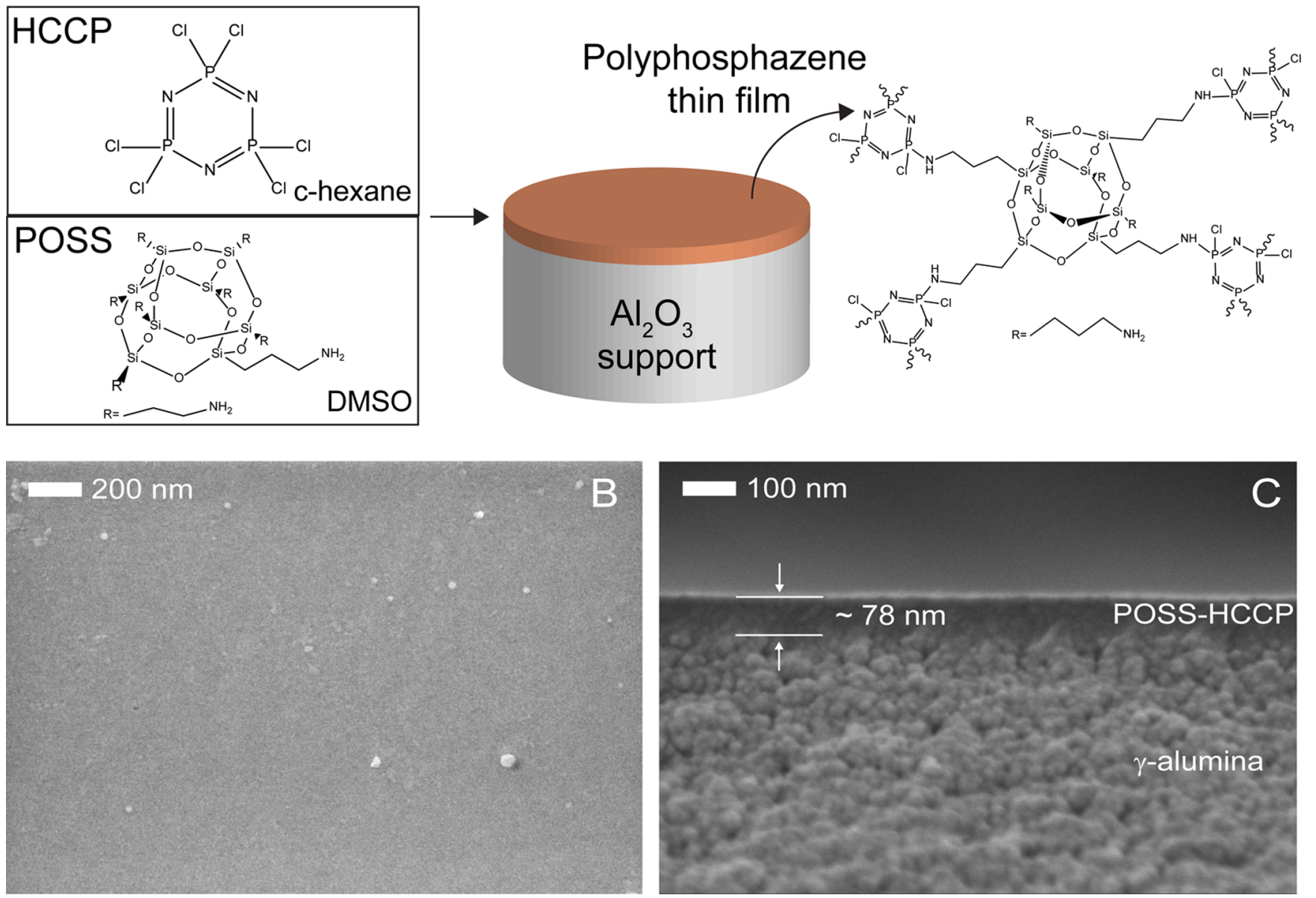

Figure 1. (A) Schematic representation of POSS-HCCP TFC membrane preparation. (B) FE-SEM picture of the POSS-HCCP membrane surface and $(\mathrm{C})$ cross-sectional FE-SEM image.

polyphosphazene membranes at higher temperatures has not been studied.

In this work, we use HCCP to prepare a membrane that can perform at elevated temperatures. This requires a highly crosslinked network with moderate macromolecular dynamics. For this purpose, a polyhedral oligomeric silsesquioxane (POSS), specifically octa aminopropyl POSS, is chosen as an eight functionalized amine monomer. This is a unique material with an inorganic cage base structure and organic functional groups that can serve as linkers. These cages can offer additional thermal and mechanical stability to the network. ${ }^{12,15}$ Previous studies by Raaijmakers et al. ${ }^{1,22}$ show that using POSS cages as the main building block in a polyimide network is beneficial to get high polymer chain rigidity for effective molecular sieving. ${ }^{11}$

To the best of our knowledge, it is the first time that the unique features of both POSS and HCCP are combined into a cyclomatrix poly(phosphazene) thin-film composite (TFC) membrane and used for gas separation at elevated temperatures. The polyphosphazene gas separation membranes are prepared through interfacial polymerization (IP) between POSS and HCCP, directly atop of a ceramic support. The formed thin crosslinked layer on top of the support permits high permeance of small molecules, such as hydrogen, combined with a low permeance of slightly larger molecules, such as methane. ${ }^{19,23}$ The polyphosphazene network is characterized for its stability, and the performance of the POSS-HCCP membranes is evaluated based on the single gas permeance and permselectivity at a wide range of temperatures.

\section{EXPERIMENTAL SECTION}

2.1. Materials. HAPS (POSS) in $n$-propanol (solids content 52.3 wt \%) was kindly provided by Funzionano AS. Phosphonitrilic chloride trimer (HCCP, 99\%) and dimethyl sulfoxide (DMSO, anhydrous, $\geq 99.9 \%$ ) were obtained from Sigma-Aldrich. Cyclohexane (EMSURE for analysis) and potassium hydroxide $(\mathrm{KOH}$, pellets extra pure) were obtained from Merck kGaA. Ethanol (EtOH, 100\% technical grade) was purchased from Boom. Porous $\alpha$-alumina discs (Ø $39 \mathrm{~mm}$, thickness $2 \mathrm{~mm}$ ) were obtained from Pervatech B.V. and used as the support.

2.2. Material Fabrication. For both the preparation of POSSHCCP freestanding films and the preparation of the TFC membranes, thin films were prepared by the IP reaction between $10 \mathrm{w} / \mathrm{v} \%$ POSS in DMSO (5:1 DMSO/0.3 M KOH) and $3.5 \mathrm{w} / \mathrm{v} \% \mathrm{HCCP}$ in cyclohexane.

2.2.1. Synthesis of POSS-HCCP Freestanding Films. POSS-HCCP freestanding films were prepared as follows. A solution of the organic HCCP solution was poured atop of the aqueous POSS solution. After $10 \mathrm{~min}$ of reaction time, a thin film was obtained that could be removed from the interface (Figure S1). The resulting solid was 
filtered, washed with water and ethanol, and dried in a vacuum oven at $50{ }^{\circ} \mathrm{C}$. The solid might also be referred to as powder.

2.2.2. Preparation of POSS-HCCP Thin-Film Composite Membranes. The porous $\alpha$-alumina supports were coated with a $3 \mu \mathrm{M}$ thick $\gamma$-alumina layer via a dip-coating and calcination process as described before by Karakiliç et al. ${ }^{24}$ This $\gamma$-alumina layer serves as an intermediate layer upon which the dense separating layer can be formed.

The TFC membranes were made by IP directly on top of the porous alumina support using an IP cell, as shown in Figure S2 (Supporting Information). First, both the POSS solution and support membrane were heated to $70{ }^{\circ} \mathrm{C}$ for $30 \mathrm{~min}$. After this, $10 \mathrm{~mL}$ of POSS solution was poured on top of the support. The cell was placed in a closed box in the oven at $80{ }^{\circ} \mathrm{C}$ for $15 \mathrm{~min}$ to ensure POSSDMSO entering the pores. After $15 \mathrm{~min}$, it was taken out of the oven, and the POSS solution was discarded. Subsequently, the membrane surface was dried by firmly applying a rubber roller three times and removing the remaining droplets with a $\mathrm{N}_{2}$ gun. The drying steps are critical to avoid delamination. Then, $10 \mathrm{~mL}$ of HCCP solution was gently poured on top. After allowing the reaction to complete for 10 $\mathrm{min}$, the solution was discarded, and the membrane surface was rinsed with ethanol. Before analysis, it was dried under air overnight, and after that, it was further dried in a vacuum oven at $50^{\circ} \mathrm{C}$ for minimum $12 \mathrm{~h}$.

2.3. Material Characterization. The thickness and morphology of the membranes were visualized using a field emission scanning electron microscope (JSM-7610F). Both the surface and cross-section of the membranes were evaluated. For the sample preparation, membranes were dried overnight in a vacuum oven at $50^{\circ} \mathrm{C}$, fractured in liquid nitrogen, and coated with a $5 \mathrm{~nm} \mathrm{Pt} / \mathrm{Pd}$ conductive layer.

The chemistry of the powders was examined by Fourier transform infrared spectroscopy in ATR mode (ATR-FTIR, PerkinElmer Spectrum Two). Spectra were collected from 16 scans with a resolution of $4 \mathrm{~cm}^{-1}$ over the wavelength range from 400 to 4000 $\mathrm{cm}^{-1}$.

X-ray fluorescence (XRF) measurements (S8 Tiger, Bruker) were performed on the synthesized powder to examine the elemental composition of the formed network.

Combined thermogravimetric analysis (TGA, STA 449 F3 Jupiler, Netzsch) and mass spectrometry (MS, QMS 403 D Aeolos MS, Netzsch) measurements were used to assess the stability of the powder upon heating $\left(10^{\circ} \mathrm{C} \cdot \mathrm{min}^{-1}, \mathrm{~N}_{2}\right.$ atmosphere $)$.

2.4. Membrane Performance. Single gas permeance experiments were carried out in the dead-end mode at a transmembrane pressure of 2 bar using a Convergence Inspector Poseidon gas permeation setup. The gases measured are as follows: $\mathrm{He}, \mathrm{N}_{2}, \mathrm{CH}_{4}$, $\mathrm{H}_{2}$, and $\mathrm{CO}_{2}$. The permselectivity was calculated as the ratio of the respective permeances. Measurements were carried out at temperatures between 50 and $250{ }^{\circ} \mathrm{C}$. Error bars represent the standard deviation between two different membrane samples.

\section{RESULTS AND DISCUSSION}

This article is divided into three parts. In the first part, the formation of TFC POSS-HCCP membranes is discussed. In the second part, the polyphosphazene networks are characterized in terms of their chemistry and thermal stability. Finally, in the third part, the gas separation performance of the POSS-HCCP membranes is evaluated.

3.1. POSS-HCCP Membrane Preparation. Figure $1 \mathrm{~A}$ gives a schematic representation of the preparation of POSSHCCP TFC membranes by the IP of POSS and HCCP on top of a ceramic support. The successful formation of thin POSSHCCP polyphosphazene films is confirmed by the SEM micrograph of the surface of the membrane in Figure 1B,C, where both surface and cross-sectional images of the TFC membrane are shown. The surface of the membrane appears smooth, confirming that a continuous and dense layer is formed on top of the $\gamma$-alumina layer. The cross-sectional image $1 \mathrm{C}$ reveals that the thickness of the layer is $\sim 80 \mathrm{~nm}$. This is a typical thickness for IP membranes. It must be noted that the spread in thickness along the sample, $\pm 40 \mathrm{~nm}$, is rather big.

3.2. POSS-HCCP Characterization. The chemistry of the formed networks is characterized by FTIR. In Figure 2, FTIR

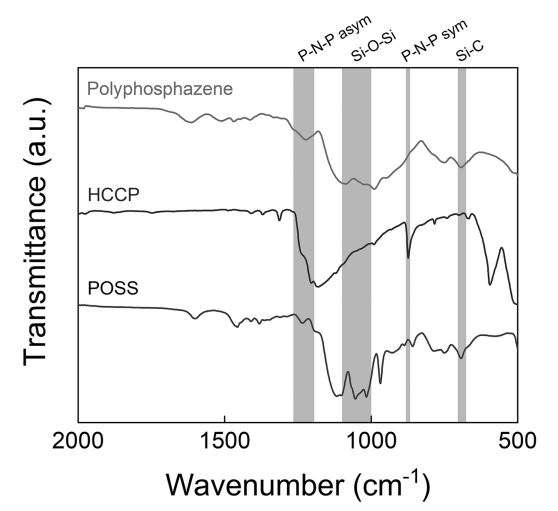

Figure 2. FTIR spectra of monomers POSS and HCCP and the formed polyphosphazene network.

spectra are shown of the used monomers, POSS and HCCP, as well of the freestanding film obtained from IP. The freestanding film shows the characteristic peaks of both HCCP and POSS. The POSS cage is apparent by the peaks in the $1000-1150 \mathrm{~cm}^{-1}$ region, ${ }^{25}$ specifically the peak at 1100 $\mathrm{cm}^{-1}$ that can be assigned to the $\mathrm{Si}-\mathrm{O}-\mathrm{Si}$ functional group. The broad peak around 1230 covers the peaks for the asymmetric P-N-P stretching of HCCP at 1238 and 1203 $\mathrm{cm}^{-1}$. Furthermore, at $875 \mathrm{~cm}^{-1}$, the peak corresponding to the symmetric stretching of $\mathrm{P}-\mathrm{N}-\mathrm{P}$ is observed. ${ }^{26}$ Finally, the peak at $690 \mathrm{~cm}^{-1}$ can be assigned to the $\mathrm{Si}-\mathrm{C}$ stretching of the POSS. From the presence of POSS and HCCP, combined with the mechanical integrity of the freestanding film, we conclude that a polyphosphazene network has been formed.

The mechanical integrity of the polyphosphazene films requires a sufficient amount of condensation reactions between POSS and HCCP, leading to a crosslinked network. The extent of crosslinking is estimated from the XRF data in Table 1,

\section{Table 1. XRF Data for the Polyphosphazene Powder}

\begin{tabular}{ccc} 
element & elemental concentration (\%) & statistical error (\%) \\
$\mathrm{Si}$ & 43.2 & 1.3 \\
$\mathrm{P}$ & 33.3 & 1.6 \\
$\mathrm{Cl}$ & 21.5 & 2.2 \\
$\mathrm{~S}$ & 2.0 & 5.9 \\
\hline
\end{tabular}

showing the elemental composition of polyphosphazene powders. The data reveal the presence of $\mathrm{Si}, \mathrm{P}, \mathrm{Cl}$, and traces of $S$ in the powder. The traces of $S$ are most likely due to the incomplete removal of DMSO from the sample. Unfortunately, $\mathrm{C}$ and $\mathrm{N}$ cannot be detected with this method.

The ratio of $\mathrm{P}$ over $\mathrm{Cl}$ is 1.5. This indicates that 4 out of the total $6 \mathrm{Cl}$ groups per HCCP molecule are involved in the reaction with the POSS cages. This implies that most of the $\mathrm{P}$ atoms are covalently attached to the network. On the other hand, the ratio of $\mathrm{Si} / \mathrm{Cl}$ is 2 , which implies two reacted HCCP molecules per POSS molecule. A similar amount of HCCP molecules per POSS cage can be deduced from the ratio of $\mathrm{Si}$ 
and $P$, which is roughly $4 / 3$. From this, it can be concluded that a crosslinked polyphosphazene network has formed in which, on average, a POSS cage is connected with four bridges.

The thermal stability of the formed network is represented by the TGA-MS data of the POSS-HCCP in Figure 3. A

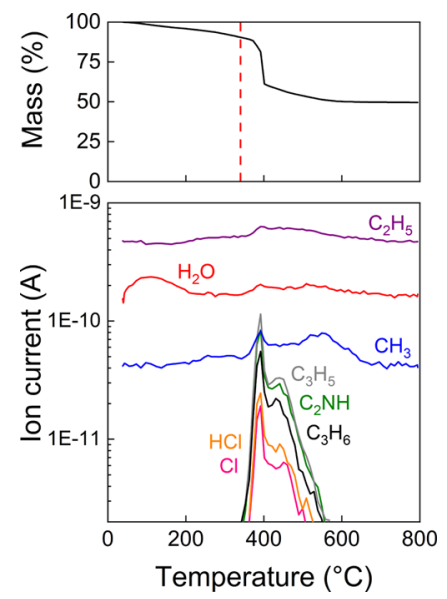

Figure 3. TGA-MS data from the polyphosphazene powder. TGA (top), selected MS data (bottom).

limited amount of mass loss is observed below $340{ }^{\circ} \mathrm{C}$. This is mainly attributed to the removal of physical bound water, also apparent from the $\mathrm{H}_{2} \mathrm{O}$ peak, but can partly be due to the loss of trapped DMSO and unreacted monomers or further crosslinking. Beyond $340{ }^{\circ} \mathrm{C}$, a sharp and substantial $( \pm 40 \%)$ decrease in mass is observed, which indicates the onset of the thermal decomposition of the network. From the
MS data, it is apparent that fragments of the propyl chain are coming off $\left(\mathrm{C}_{3} \mathrm{H}_{5}, \mathrm{C}_{2} \mathrm{H}_{6}\right.$, and $\left.\mathrm{C}_{2} \mathrm{NH}\right)$. This implies that the propyl chain is the weakest part of the network and limits the thermal stability. Besides fragments of the propyl chain, also $\mathrm{HCl}$ and $\mathrm{Cl}$ are coming off, which are expected to come from unreacted $\mathrm{Cl}$ groups on the HCCP. The remaining mass is completely governed by the cyclophosphazene and the inorganic POSS cages. The mass loss of $40 \%$ is in good agreement with the ratio of POSS and HCCP derived from the XRF data; based on this ratio, and assuming the loss of all propyl chains and unreacted chlorine groups, the mass loss can be calculated to be $41 \%$. In conclusion, the data show that the POSS-HCCP network is thermally stable up to $340{ }^{\circ} \mathrm{C}$, which is promising for use as a gas separation membrane at elevated temperatures.

3.3. Gas Permeation. Polyphosphazenes are usually classified as thermally stable polymers. ${ }^{12}$ Here, we report for the first time the gas permeance performance of POSS-HCCP polyphosphazenes at elevated temperatures.

Figure 4A shows single gas permeance data of the produced membranes as a function of gas kinetic diameter at 250, 200, 150,100 , and $50{ }^{\circ} \mathrm{C}$. The decrease in the permeance with an increasing kinetic diameter of the permeant is similar to the typical behavior observed for membranes consisting of glassy polymers. ${ }^{27,28}$ It implies that the gas molecules are permeating through a rigid molecular environment in which slightly smaller molecules have a much higher mobility (diffusion coefficient) as compared to slightly larger molecules. In contrast, for elastomers, generally the diffusion coefficient of permeants changes moderately with their size, and the permeation of larger molecules is actually larger due to their higher solubility. It is well known that the substituents of
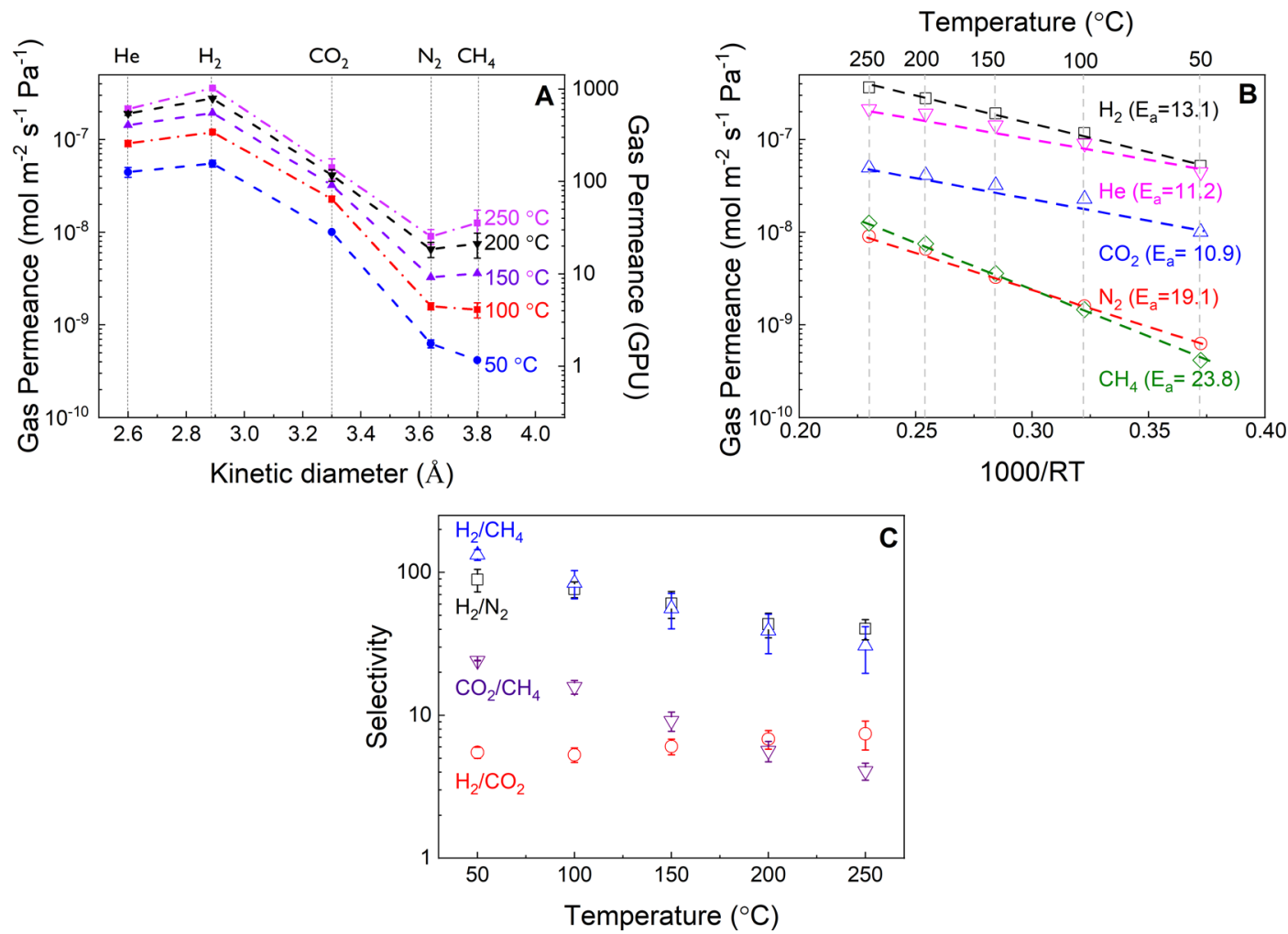

Figure 4. (A) Gas permeance as a function of gas kinetic diameter for four different temperatures. (B) Arrhenius plot of pure gas permeances. The unit of activation energies is $\mathrm{kJ} \mathrm{mol}^{-1}$. (C) Ideal selectivities of the membranes as a function of temperature. 
polyphosphazenes have a significant effect on the final properties of these polymers, ranging from elastomers to glassy polymers. ${ }^{29}$ In this case, the incorporation of the inorganic POSS cages results in moderation of the dynamics of the (hyper) crosslinked network, which is manifested by molecular sieving characteristics. It should be noted that in the polyphosphaze membranes the only pathway for diffusion is via the organic regions (the alkyl chains and the HCCP) ${ }^{30}$ because the size of the $\mathrm{Si}-\mathrm{O}$-based POSS cage is too small to accommodate even the small hydrogen molecules. Consequently, the POSS cages merely act as rigid anchoring points. The molecular sieving characteristics of the membranes at the elevated temperatures imply that the dynamics of these organic bridges are moderated. This is in agreement with earlier experimental $^{11}$ and theoretical studies. ${ }^{30}$

For all gases, the permeance increases with increasing temperature. This is expected because diffusion is generally a thermally activated process. The Arrhenius plots of the gas permeance (Figure 4B) confirm that for all gases the permeance is thermally activated, with a constant activation energy over the complete temperature range from 50 to 250 ${ }^{\circ} \mathrm{C}$. The activation energies follow the order $\mathrm{CH}_{4}>\mathrm{N}_{2}>\mathrm{H}_{2}>$ $\mathrm{He}, \mathrm{CO}_{2}$, corresponding to the variation in the kinetic diameter of the gas molecules. For larger molecules, the activation energy for diffusion is larger because more energy is required for successful jumps from one location to another in the polymer network. The exception to this order is $\mathrm{CO}_{2}$. This different behavior of $\mathrm{CO}_{2}$ is also observed for other, glassy, polymers and results from a stronger affinity of the $\mathrm{CO}_{2}$ for polar groups such as amines. The presence of these groups results in a higher solubility of $\mathrm{CO}_{2}$; solubility generally decreases with increasing temperature with an activation energy that is opposite in sign to that of the diffusion. The combined effect of temperature on solubility and diffusion is manifested by a lower "apparent" activation energy of $\mathrm{CO}_{2}{ }^{2,31}$ The values and order of the activation energies are comparable with other types of thermally stable membranes, such as polyimide and polybenzimidazole membranes. ${ }^{32}$ This implies the rigidity of the polyphosphazene network and the high gas barrier properties of these membranes.

The above results show the performance of the membranes from 250 to $50{ }^{\circ} \mathrm{C}$. However, before exposure to $250{ }^{\circ} \mathrm{C}$, the gas permeance was measured several times up to $200{ }^{\circ} \mathrm{C}$. Figure $\mathrm{S} 3$ shows the gas performance results before exposure to $250{ }^{\circ} \mathrm{C}$. After the exposure to $250{ }^{\circ} \mathrm{C}$, for all gases the permeance has increased. This implies that the exposure to 250 ${ }^{\circ} \mathrm{C}$ has resulted in a more open network. This is further evidenced by the activation energies, in Table 2 , before and after exposing to $250{ }^{\circ} \mathrm{C}$. The energies of activation have reduced, indicating a more open network. This effect is most pronounced for the larger gases, as a change in the openness of

Table 2. Comparison of Activation Energies before and after Exposure at $250{ }^{\circ} \mathrm{C}$

\begin{tabular}{lcc} 
& \multicolumn{2}{c}{ activation energy $\left(\mathrm{kJ} \mathrm{mol}^{-1}\right)$} \\
\cline { 2 - 3 } gases & \multicolumn{1}{c}{ before } & after $250^{\circ} \mathrm{C}$ exposure \\
$\mathrm{He}$ & $15.7 \pm 0.8$ & $13.1 \pm 0.7$ \\
$\mathrm{H}_{2}$ & $17.9 \pm 1.1$ & $11.2 \pm 0.4$ \\
$\mathrm{CO}_{2}$ & $23.5 \pm 2.4$ & $10.9 \pm 1.5$ \\
$\mathrm{~N}_{2}$ & $22.8 \pm 0.6$ & $19.1 \pm 0.8$ \\
$\mathrm{CH}_{4}$ & $32 \pm 1.7$ & $23.8 \pm 2.6$
\end{tabular}

the network will affect their mobility more. The exception is, again, $\mathrm{CO}_{2}$. For $\mathrm{CO}_{2}$, the reduction in activation energy is very substantial, indicating that the solubility of $\mathrm{CO}_{2}$ in the network has further increased. This can be the result of (slight) thermal degradation of the network chemistry after heating to $250{ }^{\circ} \mathrm{C}$. In particular, primary amine groups $\left(\mathrm{CNH}_{2}\right)$ would emerge due to the breaking of the weakest bond (CNHP); $\mathrm{CO}_{2}$ has a high affinity toward primary amines. ${ }^{33}$ Several thermal cycles were conducted on a fresh membrane sample to confirm these observations (Supporting Information, Figures S4-S6).

The performance of the POSS-HCCP membranes is further assessed from the permselectivities of several gas combinations. In Figure $4 \mathrm{C}$, the permselectivities of $\mathrm{H}_{2} / \mathrm{N}_{2}, \mathrm{H}_{2} / \mathrm{CH}_{4}, \mathrm{H}_{2} /$ $\mathrm{CO}_{2}$, and $\mathrm{CO}_{2} / \mathrm{CH}_{4}$ are presented as a function of temperature. Results show that at low temperatures excellent permselectivities, as high as 130 for $\mathrm{H}_{2} / \mathrm{CH}_{4}$, are obtained. The permselectivities of all the gas pairs decrease as a function of temperature, except for $\mathrm{H}_{2} / \mathrm{CO}_{2}$. This is due to differences in the activation energies of $\mathrm{H}_{2}$ and $\mathrm{CO}_{2}{ }^{32}$ As temperature increases, the $\mathrm{H}_{2}$ permeance enhances to a greater extent compared to $\mathrm{CO}_{2}$. Even at $250{ }^{\circ} \mathrm{C}$, the sieving selectivity of the membrane persists. This is due to the moderated macromolecular dynamics, resulting from the high number of connections between the organic parts of the network with the rigid silica cages, as described in the XRF section.

The performance of the membranes compare favorably with other membranes tested at the same high-temperature conditions, that is, the poly(POSS imide) membranes of Raaijmakers et al. ${ }^{11}$ At $250{ }^{\circ} \mathrm{C}$, the permselectivities of the POSS-HCCP membranes are higher than those of the poly(POSS imide) membranes; the selectivities of $\mathrm{H}_{2} / \mathrm{N}_{2}$, $\mathrm{H}_{2} / \mathrm{CH}_{4}$, and $\mathrm{H}_{2} / \mathrm{CO}_{2}$ are 40,31 , and 7.4 , respectively, while for poly(POSS imide) membranes, the selectivities are 10, 10, and 5.5 with comparable permeances. The better selectivity of the polyphosphazene membranes is due to the use of HCCP instead of the dianhydride (6FDA), leading to an augmented immobilizing effect.

In summary, the new POSS-HCCP polyphosphazene membranes are thermally stable and have persisting excellent selectivities and good permeances up to $250{ }^{\circ} \mathrm{C}$. Therefore, they have a great potential for use at high-temperature gas separation conditions, for example, for precombustion purposes.

\section{CONCLUSIONS}

In this work, we show for the first time the preparation of polyphosphazene TFC membranes via IP of POSS and HCCP. The synthesized polyphosphazene networks are highly crosslinked and display a great thermal stability, up to $340{ }^{\circ} \mathrm{C}$. The gas permeance performances of the membranes are tested at elevated high temperatures. At $250^{\circ} \mathrm{C}$, permselectivities of 40 , 31,7 , and 4 were obtained for the gas pairs $\mathrm{H}_{2} / \mathrm{N}_{2}, \mathrm{H}_{2} / \mathrm{CH}_{4}$, $\mathrm{H}_{2} / \mathrm{CO}_{2}$, and $\mathrm{CO}_{2} / \mathrm{CH}_{4}$, respectively, while maintaining permeances in the order of $10^{-7}$ to $10^{-8} \mathrm{~mol} \mathrm{~m}^{-2} \mathrm{~s}^{-1} \mathrm{~Pa}^{-1}$. At these elevated temperatures, the polyphosphazene membranes outperform other types of polymer-based membranes and are therefore very promising for the use at hightemperature applications, such as the selective separation of hydrogen in precombustion. 


\section{ASSOCIATED CONTENT}

\section{(3) Supporting Information}

The Supporting Information is available free of charge at https://pubs.acs.org/doi/10.1021/acsami.0c21968.

Freestanding film; photograph of the IP setup; gas permeation performance before exposure to $250{ }^{\circ} \mathrm{C}$; and gas permeance, activation energy, and permselectivities after different thermal cycles (PDF)

\section{AUTHOR INFORMATION}

\section{Corresponding Author}

Nieck E. Benes - Membrane Science and Technology Cluster, Faculty of Science and Technology, MESA+ Institute for Nanotechnology, University of Twente, 7500 AE Enschede, The Netherlands; 이이. orcid.org/0000-0001-9716-069X; Email: n.e.benes@utwente.nl

\section{Authors}

Farzaneh Radmanesh - Membrane Science and Technology Cluster, Faculty of Science and Technology, MESA+ Institute for Nanotechnology, University of Twente, $7500 \mathrm{AE}$ Enschede, The Netherlands; 1 orcid.org/0000-0002-93967397

Maria G. Elshof - Membrane Science and Technology Cluster, Faculty of Science and Technology, MESA+ Institute for Nanotechnology, University of Twente, 7500 AE Enschede,

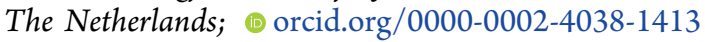

Complete contact information is available at: https://pubs.acs.org/10.1021/acsami.0c21968

\section{Author Contributions}

${ }^{\dagger}$ F.R. and M.G.E. contributed equally to this manuscript

\section{Notes}

The authors declare no competing financial interest.

\section{ACKNOWLEDGMENTS}

This work is part of the GENESIS project and the authors acknowledge the financial support from the European Union's Horizon 2020 Research and Innovation Program under the grant agreement no. 760899. This work was also partly funded by the Dutch Research Council (NWO): 〈14631 $\rangle$. We thank Funzionano AS for kindly supplying the POSS molecule as a part of the GENESIS project.

\section{REFERENCES}

(1) Koros, W. J.; Mahajan, R. Pushing the Limits on Possibilities for Large Scale Gas Separation: Which Strategies? J. Membr. Sci. 2000, 175, 181-196.

(2) Li, X.; Singh, R. P.; Dudeck, K. W.; Berchtold, K. A.; Benicewicz, B. C. Influence of Polybenzimidazole Main Chain Structure on H2/ CO2 Separation at Elevated Temperatures. J. Membr. Sci. 2014, 461, 59-68.

(3) Shan, M.; Liu, X.; Wang, X.; Liu, Z.; Iziyi, H.; Ganapathy, S.; Gascon, J.; Kapteijn, F. Novel High Performance Poly(: P -Phenylene Benzobisimidazole) (PBDI) Membranes Fabricated by Interfacial Polymerization for H2 Separation. J. Mater. Chem. A 2019, 7, 89298937.

(4) Murali, R. S.; Sankarshana, T.; Sridhar, S. Air Separation by Polymer-Based Membrane Technology. Sep. Purif. Rev. 2013, 42, 130-186.

(5) Kerry, F. G. Industrial Gas Handbook: Gas Separation and Purification; CRC Press, 2007.
(6) Staudt-Bickel, C.; Koros, W. J. Olefin/Paraffin Gas Separations with 6FDA-Based Polyimide Membranes. J. Membr. Sci. 2000, 170, 205-214.

(7) Iulianelli, A.; Drioli, E. Membrane Engineering: Latest Advancements in Gas Separation and Pre-Treatment Processes, Petrochemical Industry and Refinery, and Future Perspectives in Emerging Applications. Fuel Process. Technol. 2020, 206, 106464.

(8) Leung, D. Y. C.; Caramanna, G.; Maroto-Valer, M. M. An Overview of Current Status of Carbon Dioxide Capture and Storage Technologies. Renewable and Sustainable Energy Reviews; Elsevier Ltd, November 1, 2014; pp 426-443.

(9) Kazama, S.; Sakashita, M. Gas Separation Properties and Morphology of Asymmetric Hollow Fiber Membranes Made from Cardo Polyamide. J. Membr. Sci. 2004, 243, 59-68.

(10) Weigelt; Escorihuela; Descalzo; Tena; Escolástico; Shishatskiy; Serra; Brinkmann. Novel Polymeric Thin-Film Composite Membranes for High-Temperature Gas Separations. Membranes 2019, 9, 51.

(11) Raaijmakers, M. J. T.; Hempenius, M. A.; Schön, P. M.; Vancso, G. J.; Nijmeijer, A.; Wessling, M.; Benes, N. E. Sieving of Hot Gases by Hyper-Cross-Linked Nanoscale-Hybrid Membranes. J. Am. Chem. Soc. 2014, 136, 330-335.

(12) Rezakazemi, M.; Sadrzadeh, M.; Matsuura, T. Thermally Stable Polymers for Advanced High-Performance Gas Separation Membranes. Progress in Energy and Combustion Science; Elsevier Ltd, May 1, 2018; pp 1-41.

(13) Drioli, E.; Zhang, S.-M.; Basile, A.; Golemme, G.; Gaeta, S. N.; Zhang, H.-C. Gas Permeability of Polyphosphazene Membranes. Gas Sep. Purif. 1991, 5, 252-258.

(14) Wan, C.; Huang, X. Cyclomatrix Polyphosphazenes Frameworks (Cyclo-POPs) and the Related Nanomaterials: Synthesis, Assembly and Functionalisation. Mater. Today Commun. 2017, 11, $38-60$.

(15) Revathi, R.; Prabunathan, P.; Alagar, M. Synthesis and Studies on Phosphazene Core-Based POSS-Reinforced Polyimide Nanocomposites. Polym. Bull. 2019, 76, 387-407.

(16) Mohanty, P.; Kull, L. D.; Landskron, K. Porous Covalent Electron-Rich Organonitridic Frameworks as Highly Selective Sorbents for Methane and Carbon Dioxide. Nat. Commun. 2011, 2, 401.

(17) Chen, Y.; Li, Z.; Chen, N.; Zhang, Y.; Wang, F.; Zhu, H. Preparation and Characterization of Cross-Linked PolyphosphazeneCrown Ether Membranes for Alkaline Fuel Cells. Electrochim. Acta 2017, 258, 311-321.

(18) Gao, S.; Xu, H.; Luo, T.; Guo, Y.; Li, Z.; Ouadah, A.; Zhang, Y.; Zhang, Z.; Zhu, C. Novel Proton Conducting Membranes Based on Cross-Linked Sulfonated Polyphosphazenes and Poly(Ether Ether Ketone). J. Membr. Sci. 2017, 536, 1-10.

(19) You, M.; Li, W.; Pan, Y.; Fei, P.; Wang, H.; Zhang, W.; Zhi, L.; Meng, J. Preparation and Characterization of Antibacterial PolyamineBased Cyclophosphazene Nanofiltration Membranes. J. Membr. Sci. 2019, 592, 117371.

(20) Orme, C. J.; Klaehn, J. R.; Harrup, M. K.; Luther, T. A.; Peterson, E. S.; Stewart, F. F. Gas Permeability in Rubbery Polyphosphazene Membranes. J. Membr. Sci. 2006, 280, 175-184.

(21) Kusuma, V. A.; McNally, J. S.; Baker, J. S.; Tong, Z.; Zhu, L.; Orme, C. J.; Stewart, F. F.; Hopkinson, D. P. Cross-Linked Polyphosphazene Blends as Robust CO2Separation Membranes. ACS Appl. Mater. Interfaces 2020, 12, 30787-30795.

(22) Raaijmakers, M. J. T.; Wessling, M.; Nijmeijer, A.; Benes, N. E. Hybrid Polyhedral Oligomeric Silsesquioxanes-Imides with Tailored Intercage Spacing for Sieving of Hot Gases. Chem. Mater. 2014, 26, 3660-3664.

(23) Maaskant, E.; Gojzewski, H.; Hempenius, M. A.; Vancso, G. J.; Benes, N. E. Thin Cyclomatrix Polyphosphazene Films: Interfacial Polymerization of Hexachlorocyclotriphosphazene with Aromatic Biphenols. Polym. Chem. 2018, 9, 3169-3180.

(24) Karakiliç, P.; Huiskes, C.; Luiten-Olieman, M. W. J.; Nijmeijer, A.; Winnubst, L. Sol-Gel Processed Magnesium-Doped Silica 
Membranes with Improved H2/CO2 Separation. J. Membr. Sci. 2017, 543, 195-201.

(25) Derchi, G.; Manca, E.; Shayganpour, A.; Barone, A.; Diaspro, A.; Salerno, M. Combined Characterization of the Time Response of Impression Materials via Traditional and FTIR Measurements. Materials 2015, 8, 2387-2399.

(26) Türe, S.; Silah, H.; Tuna, M. Reinvestigations of the Reactions of Hexachlorocyclotriphosphazene with Difunctional Primary Amines Leading to Novel Dangler, Ansa and Bridged Derivatives. Spectroscopic Studies of the Derived Products. J. Mol. Struct. 2020, $1202,127232$.

(27) Omidvar, M.; Stafford, C. M.; Lin, H. Thermally Stable CrossLinked P84 with Superior Membrane H2/CO2 Separation Properties at $100{ }^{\circ}$ C. J. Membr. Sci. 2019, 575, 118-125.

(28) Japip, S.; Liao, K.-S.; Chung, T.-S. Molecularly Tuned Free Volume of Vapor Cross-Linked 6FDA-Durene/ZIF-71 MMMs for $\mathrm{H}$ ${ }_{2} / \mathrm{CO}_{2}$ Separation at $150{ }^{\circ} \mathrm{C}$. Adv. Mater. 2017, 29, 1603833.

(29) Allcock, H. R. Polyphosphazenes: New Polymers with Inorganic Backbone Atoms. Science 1976, 193, 1214-1219.

(30) Brown, D.; Neyertz, S.; Raaijmakers, M. J. T.; Benes, N. E.; Blanc, M.; Chambéry, F.-. Sorption and Permeation of Gases in Hyper-Cross-Linked Hybrid Poly ( POSS- Imide ) Networks: An in Silico Study. J. Membr. Sci. 2019, 577, 113-128.

(31) Villaluenga, J. P. G.; Seoane, B.; Hradil, J.; Sysel, P. Gas Permeation Characteristics of Heterogeneous ODPA-BIS P Polyimide Membranes at Different Temperatures. J. Membr. Sci. 2007, 305, $160-168$.

(32) Stevens, K. A.; Moon, J. D.; Borjigin, H.; Liu, R.; Joseph, R. M.; Riffle, J. S.; Freeman, B. D. Influence of Temperature on Gas Transport Properties of Tetraaminodiphenylsulfone (TADPS) Based Polybenzimidazoles. J. Membr. Sci. 2020, 593, 117427.

(33) Ko, Y. G.; Shin, S. S.; Choi, U. S. Primary, Secondary, and Tertiary Amines for CO2 Capture: Designing for Mesoporous CO2 Adsorbents. J. Colloid Interface Sci. 2011, 361, 594-602. 